\title{
Game Architecture and Virtual Teamwork
}

\author{
Esther Baldwin ${ }^{1}$, Cynthia Pickering ${ }^{2}$, David Smith ${ }^{3}$, David Abecassis ${ }^{4}$, \\ Aaron Molenaur ${ }^{5}$ \\ 1 IT Strategy, Architecture \& Innovation, Intel Corporation \\ 2 IT Collaboratory, Intel Corporation \\ 3 Qwaq Inc. \\ 4 Alloy Ventures Corporation \\ 5 Engineering Computing, Intel Corporation
}

\begin{abstract}
The panel will provide some variety to the general conference content in the sense that it represents technology solutions and experiences that are socially aware. At this point, technologies and capabilities have advanced to the point that many previous socially-oriented issues have been overcome without this necessarily being widely known. This is the point for a good conjuncture of social informatics perspectives and technological developments. The audience is strongly encouraged to pose questions from their own frameworks for the enlightenment of all, bringing together some disparate disciplines in a common conversation.
\end{abstract}

\section{Panelists' Statements}

Esther Baldwin: As corporations move towards more and more globally distributed work, they depend increasingly on tools to support remote collaboration. The basic framework for those tools today traces its origins to tools that were supplemental in nature, not central to everyday productivity. Especially as critical large-scale work artifacts are produced in virtual teams, is it important to review the basic underpinnings of how we conceptualize remote collaboration. This review must extend not just to necessary capabilities, their configuration, proportion and usability, but as well to basic beliefs and habits regarding user interfaces and technical architectures. As well, there are games now that mimic certain corporate activities in 
terms of game-like markets for transaction costs in a system. Finally, we have yet to make the full translation between gaming experience and work experience. This panel will discuss:

- A usage model for a distributed collaboration tool for design engineers working on large complex projects across the globe;

- The Miramar 3D object-oriented user interface that enables managing complexity;

- The Croquet peer-to-peer object-based game architecture that makes the new user model scalable for large projects and file sizes;

- The Serios corporate e-mail game;

- Testimony from the trenches from a software engineer who has applied his game insights to his work life.

Some of the qualities missing in existing remote collaboration tools are present in gaming: multi-tasking through objects, multi-teaming through context switching and "rooms", stimulating visuals and action environments. Neurological and heartrate research by Reeves et al, corresponding to "flow" theory of Cziktsentmihalyi, shows that limbic system and heart rate are both affected by properties of games, both visual and action-oriented. These properties can also obviously apply to productive teamwork.

Cynthia Pickering: Over a period of several years we have worked at scoping collaboration models that fit our corporate experience, using Intel technology and studies of our workforce. Tracking studies of how "virtual" Intel is, how our design engineering needs were not met by conventional tools, combined with a re-use of a context-retaining object-based 3D tool invented in the former Intel Architecture Labs have led us to the prototype tool called Miramar. We have further collaborated with Croquet developers to make Miramar scalable to a large distributed environment. The talk will present the identified needs and why we chose the solutions that we did.

David Smith: Qwaq Forums are persistent web-based virtual spaces for real collaborative work. Like offices and meeting rooms, Qwaq Forums are places where users can go to work, interact with others, share documents, chat using voice and text, and mutually identify and solve issues. Qwaq Forums are built on top of Croquet and are a high-powered, secure, behind-the-firewall solution for the distributed enterprise.

Croquet is a powerful open source software development environment that supports synchronous communication, collaboration, resource sharing and computation among large numbers of users on multiple platforms and multiple devices. The key to the system is TeaTime a replicated computation model that enables perfect sharing of even complex simulations and interactions. 
David Abecassis: Games comprise a fascinating laboratory in which both the game and player created organizations (clans, guilds, corporations) strive to capture player attention. The power of marketplaces, feedback, and reputation are applicable to the organization of information work. As the workplace becomes increasingly virtualized, businesses may more readily emulate the success of wholly virtual online games. Our first product, Attent, aims to solve the problem of overloaded inboxes by creating a synthetic economy for email.

Aaron Molenaur: Gaming software is useful as a tool for collaboration in software engineering efforts. 